\title{
Banach-Steinhaus type theorem in locally convex spaces for linear $\Sigma$-locally Lipschitzian operators
}

\author{
S. Lahrech
}




\title{
BANACH-STEINHAUS TYPE THEOREM IN LOCALLY CONVEX SPACES FOR LINEAR $\Sigma$-LOCALLY LIPSCHITZIAN OPERATORS
}

\author{
SAMIR LAHRECH
}

[Received: February 18, 2004]

\begin{abstract}
In the past, all Banach-Steinhaus type results have been established only for some special classes of locally convex spaces, e.g., barrelled spaces ([2], [3], [4]), s-barrelled spaces ([5]), strictly s-barrelled spaces ([6]), etc. Recently, Cui Chengri and Songho Han ([1]) have obtained a Banach-Steinhaus type result for linear bounded operators which is valid in every locally convex space.

In this paper we would like to prove the same result, but for linear locally Lipschitzian operators.
\end{abstract}

Mathematics Subject Classification: 47B37, 46A45

Keywords: Banach-Steinhaus theorem, locally convex spaces, linear locally Lipschitzian operators

Let $(X, \lambda)$ and $(Y, \mu)$ be locally convex spaces. Assume that the locally convex topology $\mu$ is generated by the family $\left(q_{\beta}\right)_{\beta \in I}$ of semi-norms on $Y$. Let $B\left(X_{\lambda}\right)$ denote the family of bounded set in $(X, \lambda)$ and let $\sigma \subset B\left(X_{\lambda}\right)$.

For a linear mapping $T: X \rightarrow Y$, a semi-norm $p$ on $Y$ and $C \in \sigma$, set

$$
L(p, C)(T)=\sup _{h \in C} p(T h) .
$$

Let $T: X \rightarrow Y$ be a linear operator. $T$ is said to be $\sigma$-locally Lipschitzian if

$$
\forall C \in \sigma \forall \beta \in I \quad L(\beta, C)(T) \equiv L\left(q_{\beta}, C\right)(T)<\infty .
$$

By $\operatorname{Lip}\left(X_{\lambda}, Y_{\mu}, \sigma\right)$ we denote the vector space of $\sigma$-locally Lipschitzian operators. Note that $\operatorname{Lip}\left(X_{\lambda}, Y_{\mu}, \sigma\right)$ is a locally convex space under the locally convex topology $\tau(\mu, \sigma)$ generated by the family of semi-norms $L(\beta, C), \beta \in I, C \in \sigma$.

An operator $T: X \rightarrow Y$ is said to be sequentially continuous if $\left\{x_{n}\right\}$ is a sequence in $X$ such that $x_{n} \rightarrow x$ then $T x_{n} \rightarrow T x ; T$ is said to be bounded if $T$ sends bounded sets into bounded sets. Clearly, continuous operators are sequentially continuous; sequentially continuous operators are bounded, and linear bounded operators are $\sigma$ locally Lipschitzian but in general, converse implications fail. Let $X^{\prime}, X^{s}, X^{b}$ and $X_{\sigma}^{L}$ denote the families of continuous linear functionals, sequentially continuous linear 
functionals, bounded linear functionals and $\sigma$-locally Lipschitzian functionals on $X$, respectively. In general, the inclusions $X^{\prime} \subset X^{s} \subset X^{b} \subset X_{\sigma}^{L}$ are strict.

Let $\theta\left(X, X_{\sigma}^{L}\right)$ denote the topology of uniform convergence on $\sigma\left(X_{\sigma}^{L}, X\right)$ - Cauchy sequences in $X_{\sigma}^{L}$. Note that if $\sigma=B\left(X_{\lambda}\right)$, then $X^{b}=X_{\sigma}^{L}$ and consequently, $\theta\left(X, X_{\sigma}^{L}\right)=$ $\theta\left(X, X^{b}\right)$.

Theorem 1. Let $(X, \lambda),(Y, \mu)$ be locally convex spaces and $T_{n}: X \rightarrow Y \sigma$-locally Lipschitzian operators, $n \in N$. If weak- $\lim _{n} T_{n} x=T x$ exists at each $x \in X$, then the limit operator $T$ maps $\theta\left(X, X_{\sigma}^{L}\right)$-bounded sets into bounded sets.

Proor. Let $y^{\prime} \in Y^{\prime}$. Then $\lim _{n} y^{\prime}\left(T_{n} x\right)=y^{\prime}(T x)$ for each $x \in X$. So $\left(y^{\prime} \circ T_{n}\right)_{n \in N}$ is a $\sigma\left(X_{\sigma}^{L}, X\right)$-Cauchy sequence in $X_{\sigma}^{L}$. Suppose that $B$ is $\theta\left(X, X_{\sigma}^{L}\right)$-bounded subset of $X$ and $\left\{x_{k}\right\} \subset B$. Then $\frac{1}{k} x_{k} \rightarrow 0$ in $\left(X, \theta\left(X, X_{\sigma}^{L}\right)\right)$, so $\lim _{k} \frac{1}{k} y^{\prime}\left(T_{n} x_{k}\right)=0$ uniformly in $n \in N$.

Now fix $\varepsilon>0$. There is a $k_{0} \in N$ such that $\left|\frac{1}{k} y^{\prime}\left(T_{n} x_{k}\right)\right|<\frac{\varepsilon}{2}$ for all $n \in N$ and all $k \geq k_{0}$. Fix a $k \geq k_{0}$. Since $\lim _{n} y^{\prime}\left(T_{n} x_{k}\right)=y^{\prime}\left(T x_{k}\right)$ there is an $n_{0} \in N$ such that $\left|y^{\prime}\left(T_{n_{0}} x_{k}\right)-y^{\prime}\left(T x_{k}\right)\right|<\frac{\varepsilon}{2}$. Therefore,

$$
\left|y^{\prime}\left(T x_{k}\right)\right| \leq\left|y^{\prime}\left(T x_{k}\right)-y^{\prime}\left(T_{n_{0}} x_{k}\right)\right|+\left|y^{\prime}\left(T_{n_{0}} x_{k}\right)\right|<\frac{\varepsilon}{2}+\frac{\varepsilon}{2 k}<\varepsilon .
$$

This shows that $\left\{y^{\prime}(T x): x \in B\right\}$ is bounded. Since $y^{\prime} \in Y^{\prime}$ is arbitrary, $T(B)$ is $\mu$-bounded by the classical Mackey theorem. Thus, we obtain the proof.

Theorem 1 shows that the limit operator $T$ can be bounded even if the sequence $\left(T_{n}\right)_{n}$ is not bounded.

Now we have the following useful result.

Theorem 2. For a locally convex space $X$ the following conditions are equivalent.

(1) For every locally convex space $Y$ and for every sequence $T_{n}$ of $\sigma$-locally Lipschitzian linear operators from $X$ into $Y$ such that weak- $\lim _{n} T_{n} x=T x$ exists at each $x \in X$, the limit operator $T$ is $\sigma$ - locally Lipschitzian.

(2) $\left(X_{\sigma}^{L}, \sigma\left(X_{\sigma}^{L}, X\right)\right)$ is sequentially complete.

Proof. (1) $\Rightarrow(2)$. Let $\left\{f_{n}\right\}$ be a $\sigma\left(X_{\sigma}^{L}, X\right)$ - Cauchy sequence in $X_{\sigma}^{L}$. Then, there exists a linear functional $f$ such that for every $x \in X \lim _{n} f_{n}(x)=f(x)$. Consequently, $f \in X_{\sigma}^{L}$ by (1).

(2) $\Rightarrow$ (1). Let $Y$ be a locally convex space and $\left\{T_{n}\right\}$ a sequence of $\sigma$ - locally Lipschitzian linear operators from $X$ into $Y$ such that weak- $\lim _{n} T_{n} x=T x$ exists at each $x \in X$. Let $y^{\prime} \in Y^{\prime}, C \in \sigma$. Then $\lim _{n} y^{\prime}\left(T_{n} x\right)=y^{\prime}(T x)$ at each $x \in X$. Since $y^{\prime} \circ T_{n} \in X_{\sigma}^{L}$ for all $n \in N, y^{\prime} \circ T \in X_{\sigma}^{L}$ by (2). Therefore $\left\{y^{\prime}(T x): x \in C\right\}$ is bounded and hence $T(C)$ is $\mu$-bounded by the classical Mackey theorem. Thus, $T$ is $\sigma$ - locally Lipschitzian.

Remark 1. The proof of [1, Theorem 4] seems to be incorrect and, hence, the result is not correct because even if $\lim _{n} y^{\prime}\left(T_{n} x\right)=y^{\prime}(T x)$ for each $x \in X,\left\{y^{\prime} \circ T_{n}: n \in N\right\}$ 
is not necessarily conditionally $\sigma\left(X^{b}, X\right)$-sequentially compact. Indeed, $y^{\prime} \circ T$ is not necessarily in $X^{b}$. Consequently, $y^{\prime} \circ T_{n}$ do not converge to $y^{\prime} \circ T$ in $\left(X^{b}, \sigma\left(X^{b}, X\right)\right)$.

Let $X^{\theta}$ denote the space of linear $\theta\left(X, X_{\sigma}^{L}\right)$-bounded functionals on $X$. By $\eta\left(X, X^{\theta}\right)$ we denote the topology of uniform convergence on conditionally $\sigma\left(X^{\theta}, X\right)$-sequentially compacts sets of $X^{\theta}$.

Now we have a useful proposition as follows.

Theorem 3. Let $(X, \lambda),(Y, \mu)$ be locally convex spaces and $T_{n}: X \rightarrow Y \sigma$-locally Lipschitzian operators, $n \in N$. If weak- $\lim _{n} T_{n} x=T x$ exists at each $x \in X$, then the limit operator $T$ maps $\eta\left(X, X^{\theta}\right)$-bounded sets into bounded sets.

Proof. Let $y^{\prime} \in Y^{\prime}$. Then $\lim _{n} y^{\prime}\left(T_{n} x\right)=y^{\prime}(T x)$ for each $x \in X$. It follows from Theorem 2 that $T \in X^{\theta}$. Consequently, $y^{\prime} \circ T \in X^{\theta}$. On the other hand, since $\left(y^{\prime} \circ T_{n}\right)_{n \in N}$ is $\sigma\left(X_{\sigma}^{L}, X\right)$-Cauchy sequence in $X_{\sigma}^{L}$, then $y^{\prime} \circ T_{n} \in X^{\theta}$. Therefore, $\left\{y^{\prime} \circ T_{n}: n \in N\right\}$ is conditionally $\sigma\left(X^{\theta}, X\right)$-sequentially compact.

Suppose that $B$ is a $\eta\left(X, X^{\theta}\right)$-bounded subset of $X$ and $\left\{x_{k}\right\} \subset B$. Then $\frac{1}{k} x_{k} \rightarrow 0$ in $\left(X, \eta\left(X, X^{\theta}\right)\right)$, so $\lim _{k} \frac{1}{k} y^{\prime}\left(T_{n} x_{k}\right)=0$ uniformly in $n \in N$.

Now fix $\varepsilon>0$. There is a $k_{0} \in N$ such that $\left|\frac{1}{k} y^{\prime}\left(T_{n} x_{k}\right)\right|<\frac{\varepsilon}{2}$ for all $n \in N$ and all $k \geq k_{0}$. Fix a $k \geq k_{0}$. Since $\lim _{n} y^{\prime}\left(T_{n} x_{k}\right)=y^{\prime}\left(T x_{k}\right)$ there is an $n_{0} \in N$ such that $\left|y^{\prime}\left(T_{n_{0}} x_{k}\right)-y^{\prime}\left(T x_{k}\right)\right|<\frac{\varepsilon}{2}$. Therefore,

$$
\left|y^{\prime}\left(T x_{k}\right)\right| \leq\left|y^{\prime}\left(T x_{k}\right)-y^{\prime}\left(T_{n_{0}} x_{k}\right)\right|+\left|y^{\prime}\left(T_{n_{0}} x_{k}\right)\right|<\frac{\varepsilon}{2}+\frac{\varepsilon}{2 k}<\varepsilon .
$$

This shows that $\left\{y^{\prime}(T x): x \in B\right\}$ is bounded. Since $y^{\prime} \in Y^{\prime}$ is arbitrary, $T(B)$ is $\mu$-bounded by the classical Mackey theorem. Thus, we achieve the proof.

\section{REFERENCES}

[1] Cui, Cheng Ri and Songhom, Han: Banach-Steinhaus properties of locally convex spaces, Kangweon-Kyungki Math. J., 5 (1997), No. 2, 227-232.

[2] Horváth, J.: Topological Vector Spaces and Distributions, Addison-Wesley, London, etc., 1966.

[3] Wilansky, A.: Modern Methods in Topological Vector Spaces, McGraw-Hill, Düsseldorf, etc., 1978.

[4] KöтHE, G.: Topological Vector Spaces, I, Springer-Verlag, New York, etc., 1983.

[5] SNipes, R.: S -barrelled topological vector spaces, Canad. Math. Bull. 21 (1978), No. 2, 221-227.

[6] Hsiang, W. H.: Banach-Steinhaus theorems of locally convex spaces based on sequential equicontinuity and essentially uniform boundedness, Acta Sci. Math. 52 (1988), 415-435.

\section{Author's Address}

\section{Samir Lahrech:}

Université Mohamed 1er, Faculté des sciences, Département de mathématique, Oujda, Morocco 